\title{
3-D Reconstruction and Functional Analysis of the Temporomandibular Joint
}

\author{
Reza Arbab Chirani ${ }^{1,2}$, Jean-José Jacq ${ }^{2,3}$, Christian Roux ${ }^{2,3}$, Philippe Meriot ${ }^{4}$ \\ ${ }^{1}$ Université de Bretagne Occidentale, U.F.R. d'Odontologie, Brest, France \\ ${ }^{2}$ Laboratoire de Traitement de l'Information Médicale, INSERM ERM 0102, Brest, France \\ ${ }^{3}$ Ecole Nationale Supérieure des Télécommunications de Bretagne, Brest, France \\ ${ }^{4}$ Centre Hospitalier Universitaire, service de radiologie, Brest, France \\ \{Reza.Arbab-Chirani,Philippe.Meriot\}@univ-brest.fr, \\ \{JJ.Jacq, Christian.Roux\}@enst-bretagne.fr
}

\begin{abstract}
The 3-D representation of temporomandibular joints can be performed using magnetic resonance imaging. We carried out a MRI on an asymptomatic volunteer subject. Images were reconstructed in three dimensions. This reconstruction allowed the description of the principal anatomical elements of this joint : articular disc, condyle, bilaminar region and temporal bone. A preliminary study of articular function, in the sagittal plane was also carried out with measurement of real movements of the disc.
\end{abstract}

\section{Extended Summary}

Usual 3-D reconstructions of the Temporo Mandibular Joint (TMJ) make use of two medical imaging modalities, MRI and CT scan. 2-D MRI images are used routinely to study TMJ since the eighties. Recently, some attempts have been made to access the 3 -D morphology and kinematics of TMJ $[2,3]$. In order to study articular dynamics, these representations were often associated to an extra oral movement tracking system [1]. Aims of this work is 1) to propose a 3-D reconstruction methodology of the temporomandibular joint based on MRI slices and 2) to study the function of this joint with measurement of real movements of the articular disc (translation and rotation) in the sagittal plane, during the oral opening movement.

Images of the right and left TMJ - coming from a healthy volunteer - were obtained from a 1.0 Tesla MR system, using a spin-echo sequence. The total number of slice was 90 (15 sagittal slices from each TMJ in three positions: closed jaw, halfopen jaw and completely open jaw). The $2 \mathrm{D}$ images were segmented through semiinteractive editing of the contours - the 3-D reconstruction being performed by contours stacking. Applications of such a 3-D reconstruction are numerous: representation of anatomical features, representation of articular surfaces and measurement of displacement of movable structures. From these reconstructions, the articular discs were segmented and their principal axes of inertia were computed. These axes allow an approximative measurement of real movements of the articular disc in the sagittal plane, in both translation and rotation, during jaw opening.

Three-dimensional representations, accounting for clinical reality, were obtained for the two right and left TMJ in 3 positions. Figure 1. depicts the 3-D reconstruction of the right TMJ images, in closed position. 


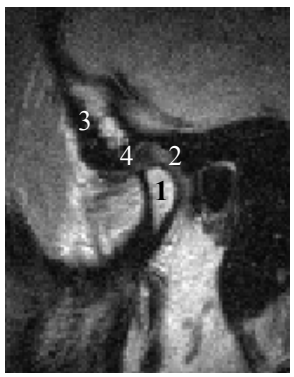

(a)

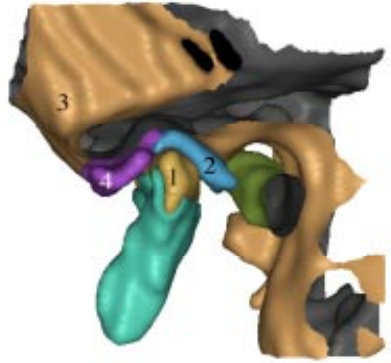

(b)

Fig. 1. Right TMJ in closed position. (a) MRI acquisition. (b) 3D reconstruction.

On the 3D representations, the inertia axes of the articular disc are represented in two different positions (Fig. 2.).

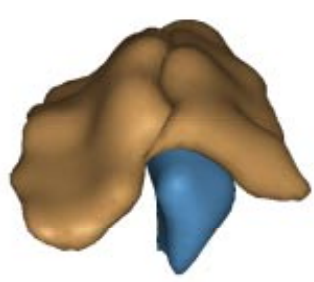

(a)

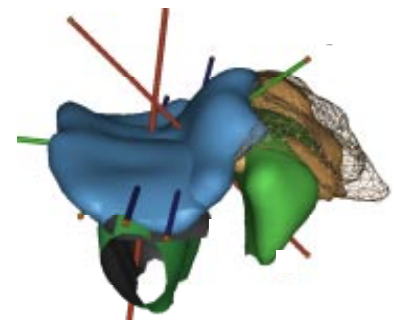

(b)

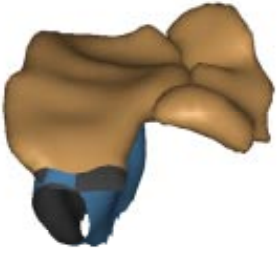

(c)

Fig. 2. Right articular surfaces. (a) Closed position. (b) Superimposition of both positions with inertia axes of the disc. (c) opened position.

The real movements for this subject has been measured. We found, for right TMJ, the following values : rotation $47^{\circ}$, translation $2.9 \mathrm{~mm}(\mathrm{X})$ and $1.6 \mathrm{~mm}(\mathrm{Y})$, which compare favorably to the literature [4]. 3-D reconstruction of the temporomandibular joint images based on MRI allows a precise anatomical description of majority of the components of this joint and constitutes a very promising way in order to study its kinematics.

\section{References}

1. Krebs M, Gallo LM, Airoldi RL. A new method for three-dimensional reconstruction and animation of the TMJ. Ann. Acad. Med. Singapore 24 (1995) 11-16

2. Motoyoshi M., Sadowsky PL. Three-dimensional reconstruction system for imaging of the temporomandibular joint using magnetic resonance imaging. J. Oral Sci. 41 (1999) 5-8

3. Price C, Connell DG, MacKay A, Tobias DL. Three-dimensional reconstruction of MRI of the temporomandibular joint by I-DEAS. Dentomaxillofac. Radiol. 21 (1992) 148-153

4. Gerber A, Steinhardt G. Dental Occlusion and the TMJ. Quintessence Publishing (1990) 